\title{
MARKOV PROCESS REPRESENTATIONS OF GENERAL STOCHASTIC PROCESSES
}

\author{
DUDLEY PAUL JOHNSON
}

\begin{abstract}
In this paper we show that any separable stochastic process on a compact metric space can be derived from a temporally homogeneous Markov process on the extreme points of a compact convex set of measures.
\end{abstract}

Let $X$ be a compact metric space with Borel field $\Sigma$. Let $T$ be either the nonnegative integers or the nonnegative rationals and let $\Omega$ be the set of all functions mapping $T$ into $X . \Omega$ with its product topology is a compact metric space and so $C(\Omega)$, the Banach space of continuous functions on $\Omega$, is separable [1, p. 340], and the weak * topology of the closed unit sphere of the Banach space rca $(\Omega)$ of regular countably additive set functions on $\Omega$ is a metric topology [1, p. 426]. If for each $\omega \in \Omega$ and $t \in T$ we define $x_{t}(\omega)=\omega(t)$ and if we let $Q$ be the $\sigma$-field of Borel subsets of $\Omega$, then for each $\mu \in \mathcal{P}(\Omega)$, the set of all probability measures in $\operatorname{rca}(\Omega)$, we get a stochastic process $X_{\mu}=\left(\Omega, Q, x_{t}, x, \mu\right)$.

If $\omega \in \Omega, \Lambda \in Q$ and $\mu \in \mathcal{P}(\Omega)$, let $\omega_{s}^{+} \in \Omega$ be defined by $\omega_{s}^{+}(t)=\omega(s+t)$, $\Lambda_{s}^{+}$be the set of all $\omega \in \Omega$ for which $\omega_{s}^{+} \in \Lambda$ and let $\lambda_{s}^{+} \in \mathcal{P}(\Omega)$ be defined by $\lambda_{s}^{+}(\Lambda)=\lambda\left(\Lambda_{s}^{+}\right)$. Let $D_{0}^{\mu}$ be the set of all $\lambda \in \mathcal{Q}(\Omega)$ which have the property that for some $0<s_{1}<\cdots<s_{n}$ in $T$ and $A_{1}, \cdots, A_{n}$ in $\Sigma, \mu\left(x_{s_{1}} \in A_{1}, \cdots, x_{s_{n}} \in A_{n}\right)>0$ and

$$
\lambda(\Lambda)=\mu\left(x_{s_{1}} \in A_{1}, \cdots, x_{s_{n}} \in A_{n}, \Lambda_{s_{n}}^{+}\right) / \mu\left(x_{s_{1}} \in A_{1}, \cdots, x_{s_{n}} \in A_{n}\right)
$$

for each $\Lambda \in a$. Let $\mathfrak{S}^{\mu}$ be the set of all weak ${ }^{*}$ compact simplexes $D$ in $\odot(\Omega)$ which contain $D_{0}^{\mu}$ and have the property that $\mu \in D$ implies that

(i) $\mu_{t}^{+} \in \mathbb{D}$ for each $t \in T$;

(ii) $\mu\left(\cdot \mid x_{0} \in A\right) \in \mathbb{D}$ for each $A \in \Sigma$. Ordering $\mathfrak{S}^{\mu}$ by inclusion and applying Zorn's Lemma, we find that $\varsigma^{\mu}$ contains minimal elements. Let $D^{\mu}$ be one of these minimal subsets of $P(\Omega)$. Let $Y^{\mu}$ be the set of extreme points of $D^{\mu}, \Omega^{\mu}$ the set of all functions mapping $T$ into $Y^{\mu}$, and $\left\{x_{t}^{\mu}, t \in T\right\}$ the family of functions mapping $\Omega^{\mu}$ into $Y^{\mu}$ defined by $x_{t}^{\mu}\left(\omega^{\mu}\right)=\omega^{\mu}(t)$. Finally, let $a^{\mu}$ be the $\sigma$-field generated by $x_{t}^{\mu}, t \in T$.

Received by the editors July 15, 1969.

AMS Subject Classifications. Primary 6040, 6060.

Key Words and Phrases. Stochastic process, temporally homogeneous Markov process, extreme points, Choquet's Theorem. 
If $\mu \in \mathcal{P}(\Omega)$ and $\lambda \in \mathcal{Y}^{\mu}$, then $\lambda_{l}^{+} \in \mathscr{D}^{\mu}$. Thus by Choquet's Theorem there exist unique measures $P^{\mu}(\cdot)$ and $P_{t}^{\mu}(\lambda, \cdot)$ on the weak ${ }^{*}$ Borel subsets of $y^{\mu}$ such that for any weak ${ }^{*}$ continuous linear functional $f$ on $\rho(\Omega)$,

$$
f(\mu)=\int_{y^{\mu}} f(\nu) P^{\mu}(d \nu) \text { and } f\left(\lambda_{t}^{+}\right)=\int_{y^{\mu}} f(\nu) P_{t}^{\mu}(\lambda, d \nu) .
$$

Let $\mu^{*} \in \mathcal{P}\left(\Omega^{\mu}, Q^{\mu}\right)$ be defined by

$$
\begin{aligned}
\mu^{*}\left(x_{t_{1}}^{\mu} \in B_{1}, \cdots,\right. & \left.\underset{t_{1}+\cdots+t_{n}}{\mu} \in B_{n}\right) \\
& =\int_{Y^{\mu}} P\left(d \nu_{0}\right) \int_{B_{1}} P_{t_{1}}\left(\nu_{0}, d \nu_{1}\right) \cdots \int_{B_{n}} P_{t_{n}}\left(\nu_{n-1}, d \nu_{n}\right) .
\end{aligned}
$$

$\mu^{*}$ is consistently defined since for any continuous linear functional $f$ on $P(\Omega)$,

$$
\begin{aligned}
\int_{y^{\mu}} f(\nu)\left(\int_{y^{\mu}} P_{s}^{\mu}(\lambda, d \xi) P_{t}^{\mu}(\xi, d \nu)\right) & =\int_{y^{\mu}} P_{s}^{\mu}(\lambda, d \xi) \int_{y^{\mu}} f(\nu) P_{t}^{\mu}(\xi, d \nu) \\
& =\int_{y^{\mu}} f\left(\xi_{t}^{+}\right) P_{s}^{\mu}(\lambda, d \xi) \\
& =f\left(\lambda_{s+t}^{+}\right) \\
& =\int_{y^{\mu}} f(\nu) P_{s+t}^{\mu}(\lambda, d \nu)
\end{aligned}
$$

and so by the uniqueness of $P_{t}^{\mu}(\lambda, \cdot)$

$$
P_{s+t}^{\mu}(\lambda, \cdot)=\int_{y^{\mu}} P_{s}^{\mu}(\lambda, d \nu) P_{t}^{\mu}(\nu, \cdot)
$$

Thus not only is $\mu^{*}$ consistently defined, but $X_{\mu}^{*}=\left(\Omega^{\mu}, Q^{\mu}, x_{t}^{\mu}, Y^{\mu}, \mu^{*}\right)$ is a temporally homogeneous Markov process with initial distribution $P^{\mu}$ and transition probability function $P_{t}^{\mu}$.

If $\mu \in \mathcal{P}(\Omega)$ and $\nu \in \mathcal{Y}^{\mu}$, then for each set $A \in \Sigma$, either $\nu\left(x_{0} \in A\right)$ or $\nu\left(x_{0} \in A^{c}\right)$ is zero. Indeed, suppose that $\nu\left(x_{0} \in A\right)>0$ and $\nu\left(x_{1} \in A^{c}\right)>0$. Then

$$
\nu(\cdot)=\nu\left(\cdot \mid x_{0} \in A\right) \nu\left(x_{0} \in A\right)+\nu\left(\cdot \mid x_{0} \in A^{c}\right) \nu\left(x_{0} \in A^{c}\right) .
$$

Since $\nu \in \mathcal{Y}^{\mu}$ and since $\nu\left(\cdot \mid x_{0} \in A\right)$ and $\nu\left(\cdot \mid x_{0} \in A^{c}\right)$ are both in $D^{\mu}$ we must have

$$
\nu(\cdot)=\nu\left(\cdot \mid x_{0} \in A\right)=\nu\left(\cdot \mid x_{0} \in A^{c}\right)
$$


Thus $\nu\left(x_{0} \in A\right)=\nu\left(x_{0} \in A \mid x_{0} \in A^{c}\right)=0$ which is a contradiction.

Let $\mathfrak{C}(\nu)$ be the class of all sets $A \in \Sigma$ for which $\nu\left(x_{0} \in A\right)>0$. Ordering $\mathfrak{C}$ by inclusion and applying Zorn's Lemma, we see that $\mathcal{C}$ has a unique minimal element which is a set consisting of a single point $\delta_{\nu}$. For each $t \in T$, we now let $\hat{x}_{t}=\delta_{x_{t}^{\mu}}$ and $\hat{X}_{\mu}$ be the stochastic process

$$
\hat{X}_{\mu}=\left(\Omega^{\mu}, Q^{\mu}, \hat{x}_{t}, X, \mu^{*}\right) \text {. }
$$

We then have the

Theorem. If $\mu \in \mathcal{P}(\Omega)$, then $X_{\mu}=\hat{X}_{\mu}$ in distribution.

Proof. Since for any continuous function $g$ on $\Omega$

$$
\begin{aligned}
\int g(\omega) \lambda_{t}^{+}(d \omega) & =\int_{y}\left(\int_{\Omega} g(\omega) \nu(d \omega)\right) P_{t}(\lambda, d \nu) \\
& =\int_{\Omega} g(\omega) \int_{y} \nu(d \omega) P_{t}(\lambda, d \nu),
\end{aligned}
$$

we have for each $\Lambda \in Q^{\mu}$

$$
\lambda_{t}^{+}(\Lambda)=\int_{y} \nu(\Lambda) P_{t}(\lambda, d \nu) .
$$

Letting $\sigma^{\mu} A=\left\{\nu: \delta_{\nu} \in A\right\}$ and dropping the superscript $\mu$ from $\mathcal{Y}^{\mu}, P_{t}^{\mu}$ and $\sigma^{\mu}$, we have for any $A \in \Sigma$ and $\Lambda \in Q$,

$$
\lambda_{t}^{+}\left(x_{0} \in A, \Lambda\right)=\int_{\sigma A} \nu\left(x_{0} \in A, \Lambda\right) P_{t}(\lambda, d \nu)=\int_{\sigma A} \nu(\Lambda) P_{t}(\lambda, d \nu) .
$$

Thus

$$
\lambda\left(x_{t} \in A, \Lambda_{t}^{+}\right)=\int_{\sigma A} \nu(\Lambda) P_{t}(\lambda, d \nu) .
$$

When $\Lambda=\Omega$,

$$
\lambda\left(x_{t} \in A\right)=\int_{0 A} P_{t}(\lambda, d \nu)
$$

and so

$$
\mu\left(x_{t} \in A\right)=\int_{y} \lambda\left(x_{t} \in A\right) P(d \lambda)=\int P(d \lambda) P_{t}(\lambda, \sigma A) .
$$

Using induction on $n$ we see that if $\lambda \in \mathcal{Y}$, then 


$$
\begin{aligned}
\lambda\left(x_{t_{1}} \in A_{1}, \cdots\right. & \left., x_{t_{1}}+\ldots+t_{n} \in A_{n}\right) \\
& =\int_{\sigma A_{1}} P_{t_{1}}\left(\lambda, d \nu_{1}\right) \int_{\sigma A_{2}} P_{t_{2}}\left(\nu_{1}, d \nu_{2}\right) \cdots \int_{\sigma A_{n}} P_{t_{n}}\left(\nu_{n-1}, d \nu_{n}\right) .
\end{aligned}
$$

Indeed if $n=1$ we have already proven it and if it true for $n=r-1$, then

$$
\begin{aligned}
& \lambda\left(x_{t_{1}} \in A_{1}, \cdots, x_{t_{1}}+\ldots+t_{r} \in A_{r}\right) \\
& =\lambda\left(x_{t_{1}} \in A_{1},\left(x_{t_{2}} \in A_{2}, \cdots, x_{t_{2}}+\ldots+t_{r} \in A_{r}\right)_{t_{1}}\right) \\
& =\int_{\sigma A_{1}} \nu_{1}\left(x_{t_{2}} \in A_{2}, \cdots, x_{t_{2}}+\cdots+t_{r} \in A_{r}\right) P_{t_{1}}\left(\lambda, d \nu_{1}\right) \\
& =\int_{\sigma A_{1}} P_{t_{1}}\left(\lambda, d \nu_{1}\right) \int_{\sigma A_{2}} P_{t_{2}}\left(\nu_{1}, d \nu_{2}\right) \cdots \int_{\sigma A_{r}} P_{t_{r}}\left(\nu_{r-1}, d \nu_{r}\right) .
\end{aligned}
$$

Thus

$$
\begin{aligned}
\mu\left(x_{0} \in A_{0},\right. & \left.x_{t_{1}} \in A_{1}, \cdots, x_{t_{1}}+\cdots+t_{n} \in A_{n}\right) \\
& =\int_{y} \nu\left(x_{0} \in A_{0}, x_{t_{1}} \in A_{1}, \cdots, x_{t_{1}}+\cdots+t_{n} \in A_{n}\right) P(d \nu) \\
& =\int_{\sigma A_{0}} \nu\left(x_{t_{1}} \in A_{1}, \cdots, x_{t_{1}}+\cdots+t_{n} \in A_{n}\right) P(d \nu) \\
& =\int_{\sigma A_{0}} P\left(d \nu_{0}\right) \int_{\sigma A_{1}} P_{t_{1}}\left(\nu_{0}, d \nu_{1}\right) \cdots \int_{\sigma A_{n}} P_{t_{n}}\left(\nu_{n-1}, d \nu_{n}\right) . \\
& =\mu^{*}\left(\hat{x}_{0} \in A_{0}, \hat{x}_{t_{1}} \in A_{1}, \cdots, \hat{x}_{t_{1}}+\cdots+t_{n} \in A_{n}\right)
\end{aligned}
$$

and the proof is complete.

\section{REFERENCES}

1. N. Dunford and J. Schwartz, Linear operators. I: General theory, Pure and Appl. Math., vol. 7, Interscience, New York, 1958. MR 22 \#8302.

2. P. A. Meyer, Probability and potentials, Blaisdell, Waltham, Mass., 1966. MR 34 \#5119.

University of California, Riverside 